\title{
Transmission dynamics of vivax malaria in Korea: effectiveness of anti-malarial chemoprophylaxis
}

\author{
Akira Endo ${ }^{*}$, Hiroshi Nishiura \\ From Challanges in malaria research: Core science and innovation \\ Oxford, UK. 22-24 September 2014
}

\section{Background}

Vivax malaria with two distinct (short- and long-term) incubation periods has been prevalent in the Republic of Korea since its re-emergence in 1993. Chemoprophylaxis has been conducted among military personnel since 1997. We estimated the time-dependent reproduction number, thereby assessing the protective effect of chemoprophylaxis.

\section{Materials and methods}

A mathematical model has been formulated using a renewal equation, estimating the yearly reproduction number (Ry) from 1993 to 2012 by maximum likelihood estimation method. We also computed Akaike Information Criterion (AIC) to test if there was a detectable change point in the trend in relation to chemoprophylaxis.

\section{Results}

Three-year average of Ry showed gradual decline through 1993-2012 with a temporary increase from 2003 to 2005, having been under the threshold 1 since 1998. AIC has suggested that the chemoprophylaxis has cut down Ry by $34 \%$ from what it would be without chemoprophylaxis.

\section{Conclusions}

The epidemic of vivax malaria in Korea has been brought under control due mainly to mass-chemoprophylaxis.

doi:10.1186/1475-2875-13-S1-P29

Cite this article as: Endo and Nishiura: Transmission dynamics of vivax malaria in Korea: effectiveness of anti-malarial chemoprophylaxis. Malaria Journal 2014 13(Suppl 1):P29.

The University of Tokyo, Tokyo, Japan 